\title{
What are the resourcing requirements for an Aboriginal and Torres Strait Islander primary health care research project?
}

\section{Sara Farnbach $h^{a, b, l}$, Graham Gee ${ }^{c, d}$, Anne-Marie Eades ${ }^{a, b}$, John Robert Evans $^{e, f}$, Jamie Fernandog, Belinda Hammond ${ }^{\text {h}}$, Matty Simms ${ }^{g}$, Karrina DeMasi', Nick Glozierj, Maree L Hacketta, b, , , on behalf of the Getting it Right Investigators}

a Neurological and Mental Health Division, The George Institute for Global Health, UNSW Sydney, Australia

b University of Sydney, NSW, Australia

c Victorian Aboriginal Health Service, Melbourne, Australia

'Murdoch Children's Research Institute, University of Melbourne, VIC, Australia

e Faculty of Health, University of Technology Sydney, NSW, Australia

+ School of Education and Social Work, University of Sydney, NSW, Australia

$g$ The Glen Centre (Ngampie), Newcastle, NSW, Australia

h Nunkuwarrin Yunti of South Australia, Adelaide, Australia

Aboriginal Medical Services Alliance Northern Territory, Darwin, Australia

j Brain and Mind Centre and Central Clinical School, University of Sydney, NSW, Australia

${ }^{k}$ University of Central Lancashire, Preston, United Kingdom

'Corresponding author: sfarnbach@unsw.edu.au

\section{Article history}

Publication date: September 2020

Citation: Farnbach S, Gee G, Eades AM,

Evans JR, Fernando J, Hammond B,

Simms M, DeMasi K, Glozier N,

Hackett ML, on behalf of the Getting it Right Investigators. What are the resourcing requirements for an Aboriginal and Torres Strait Islander primary health care research project? Public Health Res Pract. 2020;30(3):e29341911. First published 24 October 2019. https://doi.org/10.17061/ phrp29341911

\section{Abstract}

Objective and importance: To explore the role of resourcing during an Aboriginal and Torres Strait Islander primary health care research project.

Study type: Process evaluation using grounded theory approaches of a national Aboriginal and Torres Strait Islander research project $(N=500)$ named Getting it Right: the validation study.

Methods: Qualitative semistructured interviews with 36 primary health care staff and 4 community members from 9 of 10 primary health care services involved in the research project. Interviews included questions about the resources needed to conduct the research project, including flexible reimbursement to participating services (allocated within services), human resources and reimbursement to research participants (vouchers). Qualitative data were triangulated with participant feedback, study administrative data and field notes kept by the interviewer.

Results: Three themes were identified: 1) the influence of reimbursement on participating services and the research project; 2) the influence of human resources on the research project at participating services; and 3) the consequences of offering vouchers to reimburse research participants. Reimbursement was allocated to research expenses (human resources 


\section{Key points}

- Indigenous-focused primary health care research must be sufficiently funded, resourced and have adequate time allocated to be ethical and feasible

- Local decision-making processes relating to allocating research funding may maximise available funding and enhance capacity according to local priorities

- The use of participant vouchers in research requires careful, locally-based consideration. Although some staff consider that reimbursement recognises contributions by individuals and to the community, others have concerns about unintended negative consequences and logistics) or non-research expenses (service operations, equipment and conference attendance costs). Most services opted to offer vouchers to compensate participants for their time, which staff considered was appropriate recognition of participants' contributions and facilitated recruitment. Some staff described some potential unintended negative consequences from offering vouchers, including creating a welfare mentality or creating problematic expectations.

Conclusion: Primary health care research should have sufficient resourcing available, including human resource capacity, to achieve research targets. Research planning should include consideration of the existing commitments, priorities and human capacity needs of services and patients.

\section{Introduction}

Primary health care $(\mathrm{PHC})$ research can inform culturally appropriate care that contributes to the health of Aboriginal and Torres Strait Islander Peoples (hereafter referred to as Indigenous). Research should be sufficiently resourced to be feasible and ethical. ${ }^{1}$ When insufficiently resourced, securing staff time to work on the research can be challenging, potentially delaying participant recruitment and achieving research targets. ${ }^{2}$

Diversity across Indigenous communities and Indigenous-focused PHC services, (including size, funding, infrastructure and workforce) means each service may have unique resource requirements. ${ }^{3}$ Flexible and sufficient resources are needed for research to be relevant, effective and culturally respectful. ${ }^{1}$ Sufficient resourcing may also facilitate compliance with Values and ethics: guidelines for ethical conduct in Aboriginal and Torres Strait Islander health research ${ }^{4}$ (hereafter Values and ethics guideline) by funding travel that may foster ethical relationships between external researchers and communities.

There is limited information available on what constitutes sufficient research resourcing, what specific resources are required and by whom, and when and how decisions about resourcing should be made. The various approaches towards compensating research participants for their time and expenses ${ }^{5}$ indicates uncertainty about whether and how participants should be compensated. Therefore, we examined the role of resources during a National Health and Medical Research Council (NHMRC)funded, Indigenous-focused PHC-based research project named Getting it Right: the validation study ${ }^{6}$ (hereafter the research project).

The research project ${ }^{6}$ aimed to determine the validity of a previously developed ${ }^{7}$, culturally adapted depression screening tool (aPHQ-9) for use by Indigenous people. It was conducted at 10 Indigenous-focused PHC services (Aboriginal Community Controlled Health Services, Aboriginal Medical Services and a residential rehabilitation service), from six states and territories. A total of 530 Indigenous participants were recruited between 2014 and 2016. Staff were nominated by participating services to complete research activities (screen and identify potential participants, complete research interviews and complete data entry). Each participant (500/530) completed two research interviews. The protocol ${ }^{6}$ was adaptive and participating services developed local recruitment processes, while core elements of the protocol were unchanged.

Each participating service was provided resources, including flexible reimbursement to compensate for staff time. Reimbursement was provided on a per-completedparticipant basis, to allocate as participating services deemed appropriate, via the coordinating organisation (The George Institute for Global Health). Reimbursement was for a 0.5 full-time equivalent Personal Support Package level two, for 1 year (according to NHMRC standard arrangements). Resourcing also included one computer/tablet and WiFi dongle (when required) per participating service (to facilitate online data entry) and reimbursement for a $\$ 25$ food/fuel voucher per participant to local supermarkets/food stores that services could offer to participants who completed both interviews to compensate for their time. Vouchers were provided at the discretion of participating service staff and some chose to restrict vouchers from use to purchase alcohol or cigarettes (in line with organisation policies). Vouchers and resourcing were approved by the NHMRC project grant process and ethics committees.

This study aimed to explore the role of resourcing during this Indigenous-focused PHC-based research project. 


\section{Methods}

The research project ${ }^{6}$ and process evaluation methods ${ }^{8}$ have been described previously. In brief, the coordinating staff member of the research project at each participating service approached staff and community members (purposive identification) to invite them to complete qualitative semistructured grounded theory ${ }^{9}$ interviews about the research project with the lead researcher (SF) between November 2016 and June 2017 (after the research project was complete and before results were available). The interviews were conducted in a confidential setting, in person at participating services or via the telephone. The researcher conducting the interviews (SF) is a female registered nurse and $\mathrm{PhD}$ candidate who has completed training in qualitative data collection, analysis and reporting. She was project manager of the Getting it Right research project during which she developed relationships with staff and community members (1-3 years).

Process evaluation interviews were conducted using an interview guide, in three phases. Two researchers piloted the first interview guide. Interviews were transcribed verbatim. Data were managed using NVivo 10 software (Melbourne, Australia: QSR International; 2012)..$^{10}$ Independent double coding of 10 (25\%) interviews was completed by co-authors and interview reports were provided to all authors. Interview data were coded inductively to identify codes related to resourcing. A record of codes, their properties, interpretations, and feedback from authors were kept in memos, which were analysed and grouped into themes and integrated into the subsequent two interview guides. Codes were triangulated against the research project's administrative data (budgets, contracts, communication logs and ethics correspondence), participant feedback (responses to questions about the aPHQ-9 [the depression screening tool under examination] and free-text feedback collected during the research), and field notes. Process evaluation interviews continued until all potential staff or community members who wished to take part had done so. Open coding of the final two interviews identified no new codes, indicating saturation.

In this paper the terms 'Indigenous Peoples' and 'Indigenous' are respectfully used to refer to all Aboriginal and/or Torres Strait Islander Peoples of Australia.

We acknowledge the cultural diversity of Australia's Indigenous First Peoples and that they do not represent a homogenous group. ${ }^{4}$ This process evaluation was conceived, designed and conducted according to the Values and ethics guideline ${ }^{4}$, received ethical approvals (lead ethics: Aboriginal Health and Medical Research Council [1044/14], with details in the protocol) ${ }^{8}$ and was approved by participating services.
Table 1. Demographic information for staff and community members completing qualitative interviews

\begin{tabular}{lc}
\hline Staff characteristics & $\boldsymbol{N}=\mathbf{3 6}$ \\
\hline Gender & \\
Female & 24 \\
Male & 12 \\
\hline Ethnicity & \\
Indigenous & 17 \\
Non-Indigenous & 19 \\
\hline
\end{tabular}

Years working at participating service

Less than one year 0

$1-2$ years 11

2-3 years 2

3-4 years 6

$5+$ years 13

Data unavailable 4

Community member characteristics $\quad N=4$

Gender

Female 2

Male 2

Ethnicity

Indigenous

4

\section{Results}

Interviews were completed with four community members (group interview) from one community who reviewed and approved the research at their service and 36 staff (34 individually and two as a group interview) from 9 of the 10 participating services, including managers $(n=10)$, Aboriginal Health Workers (AHW) $(n=9)$, allied health staff $(n=8)$, research coordinators $(n=5)$, and general practitioners, (GPs) $(n=4)$. Between one and six staff members from the nine services participated. Staff at the tenth service chose not to participate due to staff turnover and organisational change. Approximately eight community members from one community were invited to participate (participant demographics in Table 1).

Three themes and 10 subthemes related to resourcing were identified. The themes were: 1) the influence of reimbursement on participating services and the research project; 2) the influence of human resources on the research project at participating services; and 3) the consequences of offering vouchers to reimburse research participants, and we describe these below. Descriptions of subthemes are available in Supplementary Tables 1-3, 
which are available from: www.researchgate.net/ publication/336473991_What_are_the_resourcing_ requirements

\section{The influence of reimbursement on participating services and the research project}

\section{Managers considering research involvement}

Several managers reported that they considered the reimbursement when deciding whether to become involved with the research project. Managers reported that the reimbursement was sufficient to cover resourcing for research expenses, and contributed to the participating services' financial capacity. Managers at a service where research had not previously been conducted reported:

We've knocked back a few research projects since 'cause there'd be nothing in it for us ... No staff involvement so there's no potential for upskilling ... We wouldn't have been able to do it if there wasn't money involved, it would've been a big drain on us. (Indigenous and non-Indigenous, male, managers, \#34)

Many managers considered community priorities when deciding whether to take part in the research project and reported that research focused on depression was relevant to their communities' needs.

\section{Allocating reimbursement within the service}

Some staff reported that reimbursement was allocated to research logistics or human resources (employing new staff or backfilling existing staff). When staff were hired/backfilled, recruitment targets were achieved in shorter timeframes (average 6 months) compared to when reimbursement was allocated elsewhere (average 9.5 months).

Some staff reported reimbursement was allocated to logistics that facilitated the research (e.g. funds were used to host community lunches where staff spoke with attendees about the research project).

\section{Reimbursement impacting on research conduct}

At several services, reimbursement was used to fund research-related transport expenses. Some staff reported that this dedicated transport provided flexibility to complete research interviews in an environment where patients were comfortable (at the park or their home), at ease and more likely to participate. According to these staff, participants were more honest in a nonclinical environment, which may lead to more accurate research findings. In participant feedback, 97\% reported feeling comfortable answering the questions and none provided free-text feedback about the location of the research.

Some staff reported reimbursement was used for non-research expenses (service operations, purchasing equipment and staff conference costs). Many reported this benefited the service:
We bought a[n] electric up-down bed, a really expensive one that we didn't have in our budget, so that was really good ... we halved [the money], the clinic got half and the research department got half. (Non-Indigenous, female, manager, \#27)

Some staff reported that when funds were not allocated to research, there were limited human resources available to do the research, creating pressure for staff to complete the research alongside existing duties. Sometimes access to a computer/tablet was limited or the internet connection was unstable (via the WiFi dongle provided).

\section{The influence of human resources on the research project at participating services}

\section{Human resource requirements for research}

Staff spoke of human resources as staff 'capacity' or 'availability to work on the research project'. Two managers reported considering human resources before agreeing to take part and another mentioned they would consider it more carefully in future, because it required more staff time than was originally expected. Most staff reported it took longer than expected to reach the recruitment target and some stated this was due to insufficient human resources available for the research. Review of the contracts between the participating services and The George Institute for Global Health showed that recruitment took longer than the originally contracted timeframe (3 months) at eight participating services (average 8 months).

\section{Research champions}

Many staff described 'research champions' who informally emerged and advocated for the research project both within the participating service and with patients by introducing it to the board, management and/or other staff, encouraging them to take part and advocating for it once it was underway:

I was probably one of the driving forces ... That [did the] constant reminding, chasing, finding out where we're up to. (Non-indigenous, female, manager, \#16)

Multiple staff reported advocating for the research project with patients:

I encouraged them that it was for a good cause. So this tool could be used, hopefully by GPS in the future, to help our people ... I explained what it was about and why we're part of it. (Indigenous, male, AHW, \#33)

Identifying research champions was not specified in the study protocol.

\section{Human resource challenges}

Staff reported several unexpected human resource challenges (high staff turnover, staff shortages and heavy 
workloads) and their frustrations that these contributed to delays achieving recruitment targets.

Existing research staff (with all or part of their workload allocated to research) were employed at three services. Staff perspectives varied about whether research staff should be existing or newly hired for research. Some reported that new staff could arrange logistics (reducing burden on existing staff); while others reported that existing staff with relationships with patients may make patients feel comfortable:

I'd interviewed a couple, they said they wouldn't have done it if they didn't know me 'cause... they knew me and had a relationship with me. (Non-Indigenous, female, registered nurse, \#21)

Participant feedback verified this perspective. Many reported that they preferred to complete the research with someone who they knew:

I felt comfortable answering the questions because

I was talking with someone I trusted, if it was

a stranger I would feel different. (Indigenous

participant, male, 71 years)

\section{The consequences of offering vouchers to reimburse research participants}

\section{Achieving research targets}

Eight participating services offered participants vouchers as described in the methods. Most staff reported that the vouchers facilitated recruitment, however, some identified potential unintended negative consequences, resulting in their ambivalence about voucher use as described further below.

Many staff referred to vouchers as "incentives", "thank you gifts", "rewards" or "payments". Most staff reported that vouchers facilitated participant recruitment by sufficiently acknowledging participants' contributions, time and willingness to share sensitive information:

I mean you've got to value people's time but also

... that they're prepared to talk about something

that's so personal and contribute to that research, so I think it's needed. (Indigenous and non-

Indigenous, male, managers)

Some staff reported that patients are routinely offered vouchers after annual health checks or research participation and this was problematic because it resulted in an expectation to receive a voucher after participation. Many staff reported that vouchers were valued and appreciated by participants:

A gift voucher always helps them out... They love it. Just for a \$25 gift voucher, they'll [say], 'cool, no worries.' Makes a big difference. (Indigenous, female, AHW, \#4)

Some staff considered patients were motivated to participate by the research topic, their existing relationship with the participating service or staff, or they did not expect vouchers because research was viewed as part of the services' usual program. Some staff chose not to mention the vouchers until after research interviews and reported that some participants were surprised when offered:

Some people actually turned away the vouchers, they said, 'No thanks, I didn't do it for that.' (Indigenous, male, AHW, \#5)

These staff did not specify why these participants chose to participate.

\section{Patients benefiting from participation}

A few staff suggested that vouchers were positive because they provided healthy food or financial support, and this was important because some patients had financial challenges.

\section{Considering unintended negative consequences}

Some potential unintended negative consequences from offering vouchers were reported by staff, including creating a "welfare mentality" or the vouchers setting the wrong precedent (for example, that patients will "get something" for participation), which could be harmful for future research or create the wrong motivation for $\mathrm{PHC}$ attendance. One AHW stated:

It's a slippery slope with those incentives [vouchers], maybe that's the reason why some people did the research. It's linked with that welfare mentality that's been created for our mob. Stemming back to those old ration days on the mission, it's really difficult terrain. (Indigenous, male, AHW, \#10)

\section{Ambivalence towards providing vouchers}

One AHW suggested that patients should be encouraged to attend their $\mathrm{PHC}$ and participate in research for "good health and good health of your family". Two staff described that vouchers could potentially be considered a coercion or bribe. Many staff reported ambivalence towards providing participants with vouchers for the reasons described. No participant feedback was provided about vouchers.

\section{Discussion}

As far as we are aware, this is the first research directly exploring the role of resourcing in Indigenous-focused $\mathrm{PHC}$ research. These results show that sufficient resources and time ${ }^{1}$ were available and addressed challenges that commonly arise during research (staff turnover, staff shortages and heavy workloads ${ }^{11,12}$ ), without them impacting on overall research targets other than time to complete recruitment. The flexible financial arrangements may have enhanced capacity during (by funding site-specific models to employ or backfill staff $^{1}$ ) or after the research (by funding non-research 
activities: service operations, equipment and conference attendance costs). ${ }^{4}$ When allocated to non-research expenses, the need to ensure sufficient resources were available for the research project and for open discussion with staff about these decisions was apparent.

These results demonstrate how research can build capacity when resource-allocation decisions are made at $\mathrm{PHC}$ services. Although capacity building is often a focus during Indigenous-focused research, commonly reported activities include employing staff; improving skills, capabilities or careers of Indigenous staff ${ }^{1,13}$; or developing non-Indigenous researchers' cultural competence. ${ }^{1,13,14}$ Our findings demonstrate opportunities for research to build capacity through locally driven decision-making processes. ${ }^{15}$

The spontaneous emergence of research champions as advocates demonstrates how key staff with an understanding of the 'lay of the land' can facilitate research by increasing community involvement ${ }^{1}$ and driving research. Others suggest that local research champions have local skills and expertise which increases data accuracy ${ }^{16}$ and drives data collection. ${ }^{17}$ Local champions, identified early, may facilitate research. They should be formally acknowledged for their unique skills through academic and professional avenues, such as inclusion as authors, recognition in position descriptions and/or by remuneration being provided for dedicated time for research.

The NHMRC National statement on ethical conduct in human research states that participant vouchers are acceptable to reimburse for costs. However it cautions that: "Payment ... or any other inducement that is likely to encourage participants to take risks, is ethically unacceptable". ${ }^{18}$

Although offering vouchers to participants is often reported during research ${ }^{19}$ including Indigenousfocused research ${ }^{20-23}$, to our knowledge this is the first research exploring staff perceptions of vouchers during Indigenous-focused research. Research delivering benefit is a well-established key principle during Indigenous-focused research. 1,4,18 These findings suggest that vouchers may deliver some benefit to individuals and communities. The concerns raised by staff about vouchers creating problematic expectations ${ }^{24}$ or coercing participation ${ }^{25}$ are not unique to Indigenous research. Although previous research suggests that vouchers do not create problematic expectations ${ }^{24}$, researcher training should include training about how to discuss vouchers, and ways to mitigate unintended negative consequences.

Research ${ }^{26}$ has shown that Indigenous people consider community benefit as the main motivating factor for participating in research. Our findings support for this, as some staff reported that some patients were motivated by the research topic, did not accept the vouchers, or were unaware of the vouchers until after taking part in the research. This indicates that they may have prioritised community benefit when considering participation and the vouchers did not influence their decision.
We suggest that although identifying sufficient research funding can be challenging ${ }^{2}$, it is possible within the current systems. Local decision makers should determine what resources are needed, and how they are allocated within their service and/or community, based on local priorities. Flexible resourcing may maximise resources, provide tangible benefit (in addition to benefits arising from research results) and enable PHC services to build opportunities for research champions. Recruitment may take longer than anticipated and should be planned with sufficient time to allow for unexpected delays, and funding should be provided for human resources to minimise the likelihood of delays hindering reaching research targets.

We have identified examples of how sufficient research resourcing facilitates research that addresses the Values and ethics guideline 4 (Supplementary Table 4, available from: https://www.researchgate.net/ publication/336473991_What_are_the_resourcing_ requirements) through enabling staff to modify approaches according to communities' values and aspirations (reciprocity) and potentially enhances local capacity during the research (e.g., by employing or backfilling staff) and beyond (e.g, by funding nonresearch expenses).

The lead researcher's roles as project manager of the Getting it Right research project enabled open discussions during staff interviews and improved data collection, analysis and interpretation through an in-depth understanding of the research and surrounding events. These relationships may have influenced staff to provide predominantly positive responses about the research project. With this in mind, negative responses were specifically sought from the data and are highlighted in this paper.

These findings are based on the experiences of staff, participants and community members from nine $\mathrm{PHC}$ services from communities across six Australian states and territories. We acknowledge they may not be generalisable to other Indigenous communities. Patients were not specifically asked about resourcing or vouchers and information provided in participant feedback was spontaneous.

\section{Conclusion}

This study confirms the importance of providing sufficient resourcing for research projects to enhance primary health care service, community and research capacity and to ensure the research recognises diversity and is conducted in a respectful way. Human resource capacity and the time involved with completing research should be forefront during research planning to ensure that staff have enough time to complete research activities and that research targets are achieved. The way in which resources are allocated and participants are compensated should be determined by the local 
communities where the research is being conducted, based on the human capacity needed for the research, existing workloads and other needs and priorities of services and patients.

\section{Acknowledgements}

During the completion of this work, SF was in receipt of a University of Sydney Faculty of Medicine Cross Cultural Public Health Research Award and a George Institute for Global Health John Chalmers Program Grant Scholarship; MLH was in receipt of a National Heart Foundation Future Leader Fellowship (\#100034) and an NHMRC Career Development Fellowship (Level 2 APP1141328); AME was funded by NHMRC Aboriginal and Torres Strait Islander Health Research Scholarship APP1056434 (2013-2017), UNSW Scientia Fellowship (2018-2021). Getting it Right: the validation study was supported by NHMRC grant number APP101767.

We acknowledge the participating services and participants of the Getting it Right study and this process evaluation for their contribution to this work. We also acknowledge the Getting it Right Investigators: Maree Hackett, Armando Teixeira-Pinto, Nick Glozier, Timothy Skinner, Deborah Askew, Graham Gee, Alan Cass and Alex Brown.

\section{Peer review and provenance}

Externally peer reviewed, not commissioned.

\section{Competing interests}

None declared.

\section{Author contributions}

SF lead this research including protocol development, conducting interviews and coordinating feedback from the group. JE, NG and MLH supervised SF's PhD thesis and supported protocol development and research conduct. AME, JF and GG supported protocol development. AME and GG completed data coding of transcripts. BH, MS, KDM, GG, AME, JF and JE comprise the Advisory Group. All authors contributed to the manuscript.

\section{References}

1. Jamieson L, Paradies Y, Eades S, Chong A, MapleBrown L, Morris $\mathrm{P}$, et al. Ten principles relevant to health research among Indigenous Australian populations. Med J Aust. 2012;197(1):16-8.

2. Yallop J, McAvoy B, Croucher J, Tonkin A, Piterman L. Primary health care research - essential but disadvantaged. Med J Aust. 2006;185(2):118-20.
3. Campbell MA, Hunt J, Scrimgeour DJ, Davey M, Jones V. Contribution of Aboriginal Community-Controlled Health Services to improving Aboriginal health: an evidence review. Aust Health Rev. 2018;42(2):218-26.

4. National Health and Medical Research Council. Values and Ethics: Guidelines for ethical conduct in Aboriginal and Torres Strait Islander health research. Canberra: NHMRC; 2003 [cited 2018 Oct 25] Available from: www. nhmrc.gov.au/about-us/publications/values-and-ethicsguidelines-ethical-conduct-aboriginal-and-torres-straitislander-health-research

5. Fry C, Ritter A, Baldwin S, Bowen K, Gardiner P, Holt T, et al. Paying research participants: a study of current practices in Australia. J Med Ethics. 2005;31(9):542-47.

6. Hackett M, Farnbach S, Glozier N, Skinner T, TeixeiraPinto A, Askew D, et al. Getting it Right: study protocol to determine the diagnostic accuracy of a culturally-specific measure to screen for depression in Aboriginal and/or Torres Strait Islander people. BMJ Open. 2016;6(12).

7. Brown A, Mentha R, Rowley K, Skinner T, Davy C, O'Dea K. Depression in Aboriginal men in central Australia: adaptation of the Patient Health Questionnaire 9. BMC Psych. 2013;13(1):271.

8. Farnbach S, Evans J, Eades A-M, Gee G, Fernando J, Hammond $\mathrm{B}$, et al. Process evaluation of a primary healthcare validation study of a culturally adapted depression screening tool for use by Aboriginal and Torres Strait Islander people: study protocol. BMJ Open. 2017;7(11).

9. Corbin J, Strauss A. Basics of qualitative research: techniques and procedures for developing grounded theory (Fourth edition). Thousand Oaks, California: SAGE Publications; 2015

10. QSR International Pty Ltd [Software]. NVivo 10 for Windows 2013 [cited 2015 Nov 11]. Available from: www.qsrinternational.com/product

11. Lovett R, Dance P, Guthrie J, Brown R, Tongs J. Walan Girri: Developing a culturally mediated case management model for problematic alcohol use among urban Indigenous people. Aust Health Rev. 2014;38(4):440-46.

12. Esler D, Johnston F, Thomas D, Davis B. The validity of a depression screening tool modified for use with Aboriginal and Torres Strait Islander people. Aust N Z J Public Health. 2008;32(4):317-21.

13. The Lowitja Institute. Changing the narrative in Aboriginal and Torres Strait Islander health research. Four cooperative research centres and the Lowitja Institute: the story so far. Melbourne: The Lowitja Insitute; 2017 [cited 2019 Oct 22]. Available from: www.lowitja.org. au/content/Document/Lowitja-Publishing/Changing-thenarrative_0.pdf

14. Guthrie J, Dance P, Cubillo C, McDonald D, Tongs J, Brideson T, et al. Working in partnership: skills transfer in developing a cross-cultural research team. J f Community Psychol. 2006;34(5):515-22. 
15. Brands J. The shape of things to come: Visions for the future of Aboriginal and Torres Strait Islander health research. Melbourne: The Lowitja Institute; 2014 [cited 2019 Oct 8] Available from: www.lowitja.org.au/content/ Document/PDF/the-shape-of-things-to-come.pdf

16. Kelly J, Saggers S, Taylor K, Pearce G, Massey P, Bull J, et al. "Makes you proud to be black eh?" Reflections on meaningful Indigenous research participation. Int J Equity in Health. 2012;11:40.

17. Cruz T, Davis S, FitzGerald C, Canaca G, Keane P. Engagement, recruitment, and retention in a transcommunity, randomized controlled trial for the prevention of obesity in rural American Indian and Hispanic children. J Prim Prev. 2014;35(3):135-49.

18. National Health and Medical Research Council. National statement on ethical conduct in human research. Canberra; 2007 (updated 2018) [cited 2019 Oct 8]. Available from: www.nhmrc.gov.au/about-us/publications/ national-statement-ethical-conduct-human-research2007-updated-2018

19. UyBico SJ, Pavel S, Gross CP. Recruiting vulnerable populations into research: a systematic review of recruitment interventions. J Gen Intern Med. 2007;22(6):852-63.

20. Bond C, Brough M, Spurling G, Hayman N. "It had to be my choice" Indigenous smoking cessation and negotiations of risk, resistance and resilience. Health, Risk and Society. 2012;14(6):565-81.
21. Calabria B, Clifford A, Shakeshaft A, Conigrave K, Simpson L, Bliss D, et al. Identifying Aboriginal-specific AUDIT-C and AUDIT-3 cutoff scores for at-risk, highrisk, and likely dependent drinkers using measures of agreement with the 10-item Alcohol Use Disorders Identification Test. Addict Sci Clin Pract. 2014;9:17.

22. Carey T. A qualitative study of a social and emotional well-being service for a remote Indigenous Australian community: implications for access, effectiveness, and sustainability. BMC Health Serv Res. 2013;13(80).

23. Lee K, Harrison K, Mills K, Conigrave K. Needs of Aboriginal Australian women with comorbid mental and alcohol and other drug use disorders. Drug Alcohol Rev. 2014;33(5):473-81.

24. Singer E, Van Hoewyk, Maher MP. Does the payment of incentives create expectation effects? Public Opinion Quarterly. 1998:152-64.

25. Wertheimer A, Miller FG. Payment for research participation: A coercive offer? J Med Ethics. 2008;34(5):389-92.

26. Guillemin M, Gillam L, Barnard E, Stewart P, Walker $H$, Rosenthal D. "We're checking them out": Indigenous and non-Indigenous research participants' accounts of deciding to be involved in research. IntJ Equity Health. 2016;15(1):8.

\section{Copyright: (c) (i) (2)}

(C) 2019 Farnbach et al. This article is licensed under the Creative Commons Attribution-NonCommercial-ShareAlike 4.0 International Licence, which allows others to redistribute, adapt and share this work non-commercially provided they attribute the work and any adapted version of it is distributed under the same Creative Commons licence terms. See: www.creativecommons.org/licenses/by-nc-sa/4.0/ 